\title{
A MULTI-CRITERIA DECISION SUPPORT SYSTEM FOR THE FORMATION OF COLLABORATIVE NETWORKS OF ENTERPRISES
}

\author{
José António Crispim \\ Universidade do Minho, PORTUGAL \\ crispim@eeg.uminho.pt \\ Jorge Pinho Sousa \\ INESC Porto, Faculty of Engineering Univ. Porto, PORTUGAL \\ jsousa@inescporto.pt
}

\begin{abstract}
In this paper we present a Decision Support System (DSS) to deal with the partner selection problem taking place in the formation or re-organization of a Virtual Enterprise (VE). This DSS is based on a multi-criteria model and handles several types of data (numerical, interval, linguistic and binary). This approach is used to facilitate the expression of the decision maker's preferences and assessments about the potential partners and can be performed individually or by group. The system also allows the assignment of a degree of confidence to each linguistic statement. The operation of the DSS is structured in two phases. In the first phase it determines the set of nondominated alternatives (potential VEs) through the use of meta-heuristics. The second phase ranks the alternatives for a possible network of enterprises configuring the VE. This is achieved through a procedure based on linguistic analysis and distance measures.
\end{abstract}

\section{INTRODUCTION}

In the information age competition is expected to take place less among single companies, and increasingly among groups of enterprises working together to explore emerging business opportunities (Katzy and Dissel, 2001). This new reality is in fact giving smaller companies the possibility to compete with large multinationals. A Virtual Enterprise (VE) is a temporary alliance between globally distributed independent companies working together to improve their competitiveness by sharing resources, skills, risks and costs. Since each partner brings a strength or core competence to the consortium, the success of the VE strongly depends on the achieved level of co-operation. The creation of a VE is usually triggered by a market opportunity, giving rise to a "project". Projects are usually decomposable in relatively independent sub-projects or activities. The work needed to "fulfil" a project involves a set of collaborative activities that can be dependent, when relations of precedence between activities exist, or independent, if that is not the case. Therefore, before a VE is formed, the different input and output characteristics of each activity have to be clearly defined, particularly the required degree of synchronisation, the actors involved and the resources needed.

The success of a project is strongly dependent on the composition of the VE. Hence, the selection of the partners becomes a crucial part of its lifecycle. This 
phase is even more important when, due to the multiplicity of opportunities the dynamic environment can create, the VE has to be formed very quickly in order to meet deadlines or when an enterprise can participate in various VEs at the same time. The problem of partner selection also arises when the VE needs to be reorganized by adding/expelling some members or by re-assigning tasks or roles in order to better cope with new market circumstances. During the VE formation, different obstacles can arise such as lack of a culture of cooperation, lack of trust, existence of different behaviours, different perceptions of the environment and different (and even competing) priorities and motivations. To overcome these obstacles, Camarinha-Matos and Afsarmanesh (2003) suggest the creation of breeding environments (BE) consisting of long-term networked structures with common infrastructures, common ontologies, and mutual trust.

In this paper we present a Decision Support System (DSS) to deal with the partner selection problem under a multi-criteria perspective, where several types of information (numerical, interval, linguistic and binary) are allowed, in order to facilitate the expression of the decision maker's preferences or assessments about the potential partners, in an individual or group decision making scenario. This is in practice an important requirement as the multiplicity of factors considered when selecting partners for a business opportunity, such as cost, quality, trust and delivery time, cannot be expressed in the same measure or scale.

The DSS starts by considering that all companies (the potential partners) are present in a network (the breeding environment), interacting with each other. However, it is assumed that not all pairs of companies are linked. The main objectives of the DSS are to facilitate the rapid VE formation (or re-organization), and to improve the informal cooperation between the enterprises of the network through the simulation of alternative operating scenarios. The DSS also handles the existence of several VE formation processes inside the network, simultaneously or at different moments. Its operation is structured in two phases. In the first phase it determines the set of non-dominated alternatives (potential VEs) through the use of metaheuristics. The second phase ranks the alternatives for a possible network of enterprises, thus configuring the VE. This is achieved through a procedure based on fuzzy clustering, linguistic analysis and distance measures.

The remainder of the paper is organized as follows. In Section 2 the problem is described, in Section 3 the DSS is presented, in Section 4 an illustrative example is provided and finally, in Section 5 some preliminary conclusions are presented.

\section{PROBLEM DESCRIPTION}

The VE formation process can be described in the following way. Assume a network $A$ representing all potential partners (companies) and their relationships. Companies and links are characterised by a set of $m$ attributes, some assigned to the nodes and some assigned to the edges of the network. These attributes will be used to define the criteria for evaluating solutions (i.e. VE configurations). The first step in this modelling process is to carefully define what attributes are going to be considered on both subsets. The Decision Maker can weight the attributes according to his believes about their relative importance to the project under consideration.

The network includes a set of $n$ companies connected with each other, capable of handling activities and of providing a finite amount of resources, available over 
specific intervals of time. Two types of connections between enterprises are allowed: a) a direct relation exists when a pair of companies has had a previous commercial relation; b) assume that enterprise $J$ has a direct relation with $I$, and $I$ has a direct relation with $K$, then an indirect relation exists between $J$ and $K$.

Moreover assume project $P$ involving $k$ activities that demand a specific amount $Q$ of resources and have to be performed within a determined interval of time $S$.

The partner selection problem consists of choosing the best group of companies to perform all $k$ activities of project $P$ taking into account a set of evaluation criteria based on the $m$ attributes established for the network. The main constraints of the problem are time windows limitations and the minimum amount of resources required.

Partner selection can be viewed as a multiple attribute decision making (MADM) problem (Li and Liao, 2004), i.e. a process where the DM must choose from a set of limited alternatives that are typically defined explicitly in terms of attributes. In this problem, the alternatives correspond to groups of enterprises that have the resources and skills needed to carry out the project. Infeasibility is not considered, i.e. groups of enterprises that are not able to carry out the project are not accepted. The DM's preferences are often used to rank alternatives, with the best alternative being usually selected by comparisons through the various attributes that are often hard to quantify (Pohekar and Ramachandran, 2004). In this work the DM has the possibility to use different types of variables (numerical, linguistic, interval and binary) to express his preferences. Given the multi-criteria nature of the problem, there is generally no "optimal" alternative, and a good compromise solution must therefore be identified. Figure 1 gives an overall idea about the VE formation problem, namely the network, the companies, the relationships and the groups of enterprises that are potential VEs.

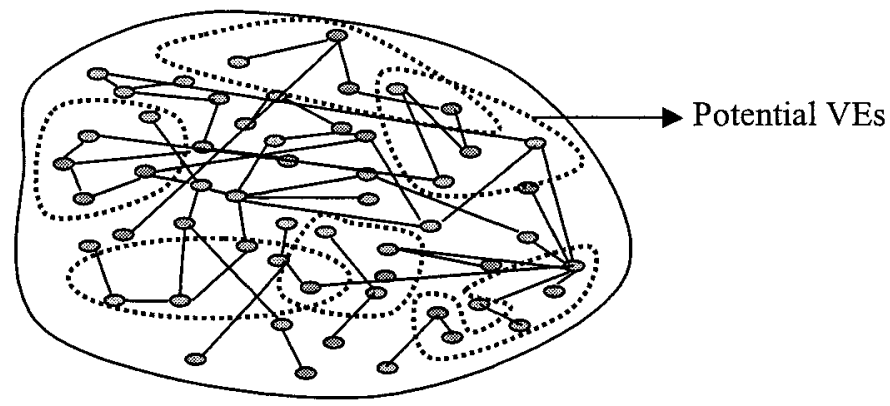

Figure 1: Multiple potential VEs within a network

\section{THE DECISION SUPPORT SYSTEM}

The DSS briefly described in this paper follows a simple architecture (figure 2) that comprises an interface with the DM, a data base, an output report area and three "functional" modules: the generation module, the ranking module and the simulation module. Its operation follows a 2-phase approach consisting of running the generation module first, and then the ranking module. The simulation module is an 
auxiliary module of the ranking module and is used to determine weight vectors for the attributes by Monte Carlo simulation.

This architecture aims at: i) supporting the $\mathrm{DM}$ in structuring the problem through the definition of the project (Goals that are to be achieved by performing Activities, requiring Skills and Competencies, developed by Enterprises described by their attributes and by the attributes of their relationships); ii) generating a ranking of potential VEs capable of performing the project (market opportunity); iii) providing support for "what if" analysis; and iv) analysing, in a given time horizon, how the network reacts when various projects take place, simultaneously or not. The DSS supports the existence of different types of data, individual and group DMs, and allows the DM to choose the number, kind and type of attributes to use in order to describe the network and the solution. Next, we will briefly describe each element of the DSS that was implemented in $\mathrm{C}++$.

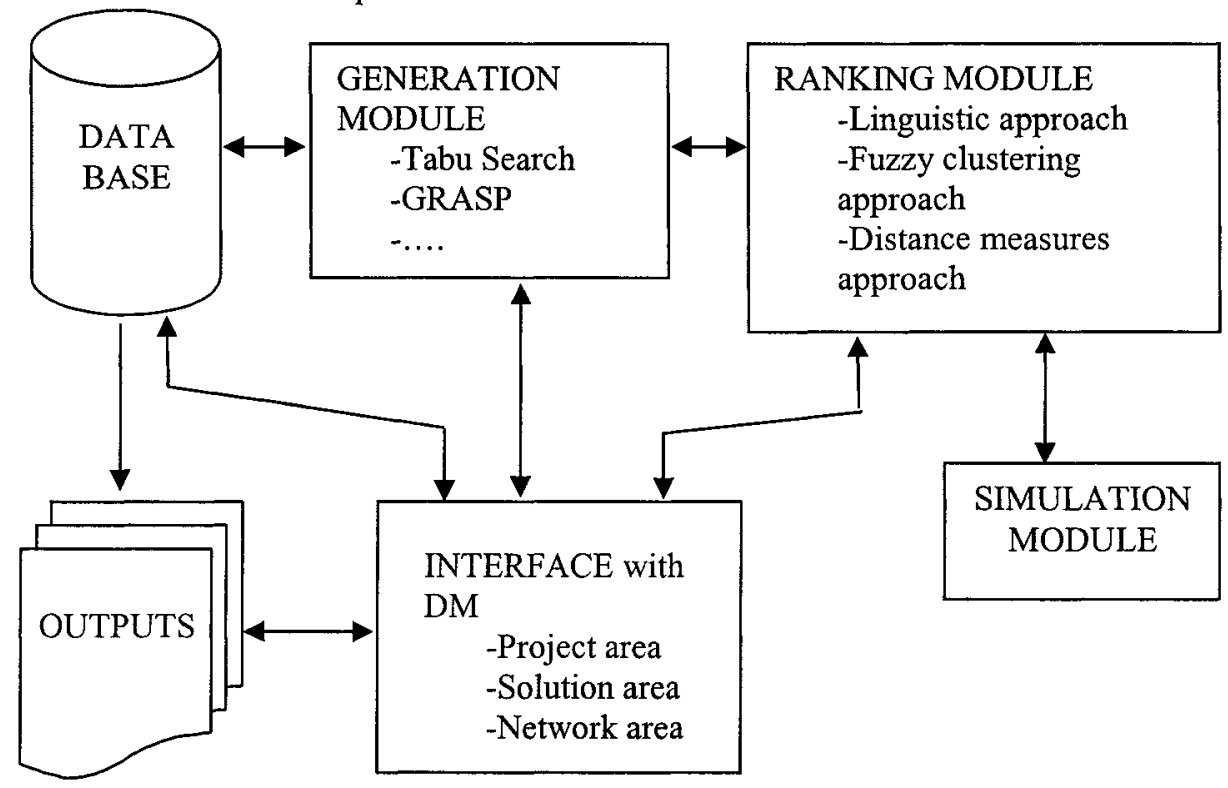

Figure 2: Architecture of the DSS

\subsection{Interface}

The DSS interface is formed by three distinct areas (Project area, Network area, and Solution area) and allows the introduction of data by text files or directly by the keyboard. The Project area is used to introduce information about the business opportunity being analysed, i.e. information about its activities (what activities, if there are precedences in their execution, execution interval times and quantities involved). The Network area is used to define the attributes that characterise the enterprises (nodes) and their relationships (arcs, edges). The Solution area is used to introduce information about the attributes used to compare and evaluate groups of enterprises (potential VEs). These attributes can be those considered in the Network area, all or only some of them, or they can be the result of an expression/combination of the Network area attributes. The Solution area can also 
be used to introduce constraints such as time windows and to introduce weights or to impose thresholds for trust, risk, etc.

\subsection{Generation Module}

This module is built around some metaheuristic algorithms, used to find a set of non-dominated alternatives to perform each project. Metaheuristics are approximate methods designed to solve hard combinatorial optimisation problems (Reeves, 1993). A metaheuristic is an iterative generation process that guides a subordinate heuristic while exploring the search space. It combines sophisticated rules to search different neighbourhood structures, memory structures and learning strategies in order to efficiently find near-optimal solutions (Osman and Kelly, 1996). The most well known metaheuristics are probably "simulated annealing" and "tabu search".

By repeatedly running these algorithms, it is possible, for the same project, to generate a large set of diverse solutions according to the type and number of attributes considered. However this set should also be small enough to be treatable and understandable by the DM. Moreover, it should cover the entire "solution curve", i.e. contain solutions that represent well the different possible compromises between the attributes. This can limit the number of alternatives to be kept by the system, leading to the deletion of the most similar ones.

In this work, we have implemented a Tabu Search (TS) metaheuristic (see e.g. Glover, 1989, 1990, 1993 and Glover and Laguna 1996, 1997). By a memory mechanism, TS is able to forbid certain movements during the search process, in order to diversify it. To do this, it stores the recently accepted solutions or attributes of these solutions in memory (tabu list) in order to prevent cycling. The main competitive advantage of Tabu Search with respect to alternative heuristics, also based on local search, is the intelligent use of the past history of the search to influence its future steps.

In our problem, a solution is represented by a set of companies in the network associated to the different activities, along with the values of the corresponding attributes.

The initial solution is generated through a simple process consisting of the following steps:

- Create a table of enterprises, activities and constraints (e.g. capacities). A given activity may be performed by a group of enterprises if, for example, separately they do not have enough resources. In this case, the group of enterprises is added to the network as a single unit and the attribute values that describe it result from the attribute values of the different enterprises.

- From that table randomly select one enterprise or a group of enterprises capable of handling the first activity of the project.

- Search, for the next activity, the enterprises with direct relations with the enterprise previously chosen and select the one which has the higher value for one or a group of attributes (for example, the higher value of trust). If no direct relations exist, search for an indirect relation and, if it does not exist, search for any other feasible enterprise.

- Repeat the previous step until all the activities of the project have been assigned to enterprises. If there is an activity not performed by any enterprise, ask the DM to introduce a new enterprise in the data base in order to overcome this problem. 
The improvement of a solution is done by local search, where the neighbourhood structure consists of swapping an enterprise in the solution with an enterprise outside the solution. In this way, the search starts by exploring an enterprise that can do the first activity. If this replacement leads to a non-dominated alternative, this new set of enterprises is saved in the table of alternatives. The activities are explored by the order they have been defined in the project. Two tabu lists are used, the first forbids the utilization of the enterprises recently chosen, and the second forbids the choice of the last activity selected. This exploration of the neighbourhood structure is repeated until the search cannot achieve any alternative solution during 5000 consecutive iterations.

The strategy used in the neighbourhood search is similar to the first improvement and only accepts feasible solutions. An intensification strategy is adopted after a given number of consecutive dominated solutions is found and consists of re-starting the procedure with one of the non-dominated solutions saved in the table of alternatives randomly chosen. The algorithm only saves those non-dominated solutions that are significantly different. For that purpose, we consider an "indifferent zone attribute value" which consists of admitting that the attribute values are similar if their difference in percentage is smaller than a given value ( $\pm 5 \%$, for example). This percentage is provided by the DM, and can be different for each attribute.

\subsection{Ranking Module}

\subsubsection{Linguistic Approach}

There are decision situations in which the information cannot be assessed precisely in a quantitative form, due to its nature (e.g. trust) or because either information is unavailable or the cost of its computation is too high. In these situations an "approximate value" may be acceptable and so the use of a linguistic approach is appropriate (Herrera et al., 2004). "Linguistic variables" will represent qualitative aspects, with values that are not numbers but words or sentences in a natural language, thus making it easier to express preferences.

For our DSS we have adopted a 2-tuple fuzzy linguistic representation model (see Herrera and Martinez, 2000) as it allows the management of non-homogeneous information (numerical, linguistic interval, binary). This model is based on symbolic methods and takes as the base of its representation the concept of Symbolic Translation. It represents the linguistic information by means of a 2-tuple $(s, \alpha)$ where $s$ is a linguistic term and $\alpha$ is a numerical value with $\alpha \mathrm{C}[-0,5 ; 0,5]$, representing the original result translated to the closest index label in the linguistic term set.

In the linguistic decision analysis of an MCDM problem, the solution scheme should involve the following three steps (Herrera and Herrera-Viedma, 2000): the choice of the linguistic term set with its semantic (meaning), the choice of the aggregation operator of linguistic information, and the choice of the best alternatives. The linguistic term set, usually called S, comprises a set of linguistic values that are generally ordered and uniformly distributed. For example, a set $\mathrm{S}$ of seven terms could be given as follows: $S=\left\{s_{0}=\right.$ none; $s_{1}=$ very low; $s_{2}=$ low; $s_{3}$ $=$ medium; $\mathrm{s}_{4}=$ high; $\mathrm{s}_{5}=$ very high; $\mathrm{s}_{6}=$ perfect $\}$, in which $\mathrm{s}_{\mathrm{a}}<\mathrm{s}_{\mathrm{b}}$ if $\mathrm{a}<\mathrm{b}$. The semantics of the elements in the term set (the meaning of each term set) is given by fuzzy 
numbers defined on the $[0,1]$ interval and described by membership functions. Since the linguistic assessments given by the individuals are approximate, because it may be impossible or unnecessary to obtain more accurate values, Delgado et al. (1998) and Herrera et al. (2002) consider that trapezoidal or triangular membership functions are good enough to capture the vagueness of those linguistic assessments. In our DSS we have adopted triangular membership functions and accept different cardinalities for $\mathrm{S}$, and different semantics in the term set, depending on the DM and/or the attribute in question. This becomes an advantage of the DSS because it allows the DM to be more or less detailed, when in presence of distinct attributes. For example, for "trust" use the term set $S=\left\{s_{0}=\right.$ none; $s_{1}=$ very low; $s_{2}=$ low; $s_{3}$ $=$ medium; $\mathrm{s}_{4}=$ high; $\mathrm{s}_{5}=$ very high; $\mathrm{s}_{6}=$ total $\}$ and for "prestige" $\mathrm{S}=\left\{\mathrm{s}_{0}=\right.$ none; $\mathrm{s}_{1}$ =medium; $s_{2}=$ total $\}$.

The 2-tuple approach implemented follows a scheme composed by three phases: unify the information into fuzzy sets over a basic linguistic term set (BLTS), aggregate them in a decision matrix, and rank the alternatives. Our implementation of this technique is slightly different from the original since we allow multiple attributes and eventually multiple DMs. In this way, to unify information, first, we have to aggregate (through a simple arithmetic mean) the attribute values of each enterprise. This aggregation can be complemented by attribute weights provided by the DM or by running the simulation module. After that, we transform the mixed information into fuzzy sets over a BLTS that corresponds to the term set with more linguistic values. Then, a transformation of the aggregate fuzzy sets into 2-tuples (s, $\alpha$ ) is performed. Finally the ranking is achieved by calculating the dominance degree.

\subsubsection{Distance Measures}

Fuziness is inherent to most decision making processes when linguistic variables are used to describe qualitative data. Since linguistic labels, such as "very high", to describe the "trust" linguistic variable can be represented using positive triangular fuzzy numbers, a new approach to rank alternatives based on distance calculations can be used. This approach consists in comparing fuzzy numbers through the use of a fuzzy distance measure computed between each alternative and a crisp ideal solution (Tran and Duckstein, 2002) or a fuzzy positive ideal solution (Li and Yang, 2004; Ding and Liang, 2004). According to Tran and Duckstein (2002), the ranking order of all alternatives is obtained by comparing the distances of each alternative to two points, the crisp maximum and the crisp minimum, and the alternative first rank has the smallest distance to the maximum and the highest to the minimum. These two points of reference could be predetermined targets. Therefore in the Distance measures area we calculate the distances between each attribute value in the vector solution and its associated ideal maximum and minimum (values defined in the database for each attribute or introduced by the DM). For linguistic variables, we can use the distance between two triangular fuzzy numbers (the membership functions used in this DSS), presented by Li and Yang (2004), identical to the euclidean distance. In the case of interval values, we follow the distance formula presented by Tran and Duckstein (2002). In the case of numerical values, we use the distance between real numbers ( $\mathrm{Li}$ and Yang, 2004). The main problem here is how to aggregate in a single value the non-homogeneous distance values we obtain, an issue that the previous studies do not take into consideration. To overcome this 
problem, after determining the distances, a normalization is performed and therefore all the distances are in the $[0,1]$ interval. Then an average is calculated and a final list, ranking the alternatives, is presented to the DM.

\subsection{Simulation Module}

This module is used when the DM wants to weight attributes in order to express their relative importances but is only capable of providing their rank order, or if the DM desires to perform some sensivity analysis on the weights. In this case, the module randomly computes weights for each attribute respecting the attributes rank order introduced and calculates the new alternative ranking. This process is repeated for a number of iterations (5000). A matrix containing the alternatives (rows) and the positions they assume (columns) is used to count every new alternative hierarchy. At the end a final alternative ranking is obtained according to the matrix information (for example, alternative $D$ is the "best" because it occupies the first position 3500 times, the second 1400 times, ...). The simulation process, similar to that described by Butler et al. (1997), can be summarized as follows:

1. Repeat until stop criterion is satisfied

- Generate n-number attributes random weights $\left(w_{i}\right)$ on the interval $[1,99]$.

- Normalize the weights, with $0<w_{i}<1, \forall i$, and $\sum_{i=1}^{n} w_{i}=1$.

- Order the weights and assign them to the attributes respecting the attributes hierarchy provided by the DM

- Through the use of the additive model, compute the new alternative hierarchy.

- Save this new hierarchy in a matrix of results.

2. Obtain the final rank order list of alternatives according to their counter.

\section{ILLUSTRATIVE EXAMPLE}

Assume we would like to form a VE to perform a project decomposed in 5 activities as shown in Table 1.

Table 1: Project data

\begin{tabular}{|c|c|c|c|c|c|}
\hline $\begin{array}{c}\text { Activities } \\
\text { (code) }\end{array}$ & $\begin{array}{c}\text { Precedent } \\
\text { activities }\end{array}$ & $\begin{array}{c}\text { Standard } \\
\text { duration }\end{array}$ & $\begin{array}{c}\text { Earliest } \\
\text { start } \\
\text { time }\end{array}$ & $\begin{array}{c}\text { Latest } \\
\text { finish } \\
\text { time }\end{array}$ & $\begin{array}{c}\text { Quantity } \\
\text { of } \\
\text { resources }\end{array}$ \\
\hline 8 & - & 24 & 111 & 171 & 121 \\
\hline 4 & - & 9 & 101 & 160 & 181 \\
\hline 5 & - & 35 & 28 & 295 & 146 \\
\hline 6 & 4 & 48 & 160 & 298 & 280 \\
\hline 3 & 4 & 47 & 160 & 228 & 62 \\
\hline
\end{tabular}

Suppose a network composed by 100 companies described by a set of numerical variables (activities that each enterprise can execute, its capacity considered in terms of an available quantity, years of existence), an interval variable (time window during which the resources are available), and some linguistic variables (global 
performance, dimension, and management prestige). There are direct and indirect connections between the companies, comprising information such as distance, capacity of the link (in quantity), cost, price, trust and risk. For the linguistic variables we assume triangular membership functions with a cardinality of 5 with the correspondent term set \{none, low, more or less, high, perfect\}. The values of indirect connections are estimated as half of the direct connections. Attributes have an equal weight and there is a single DM. By applying the tabu search procedure, we have obtained the non-dominated alternatives set shown in table 2.

Table 2: Non-dominated alternatives data

\begin{tabular}{|c|c|c|c|c|c|c|c|c|c|c|c|c|c|}
\hline \multirow[b]{3}{*}{ VE1 } & \multicolumn{5}{|c|}{ company num ber } & \multirow{2}{*}{\multicolumn{2}{|c|}{ price interval }} & \multirow[b]{2}{*}{ cost } & \multirow[b]{2}{*}{ distance } & \multirow[b]{2}{*}{ trust } & \multirow[b]{2}{*}{ prestige } & \multirow[b]{2}{*}{ risk } & \multirow{2}{*}{$\begin{array}{c}\text { global } \\
\text { performance }\end{array}$} \\
\hline & Act 1 & Act2 & Act3 & Act4 & Act5 & & & & & & & & \\
\hline & 74 & 9 & 13 & 22 & 47 & 9 & 48 & 4564 & 193 & ML & $\mathrm{P}$ & $\mathrm{ML}$ & ML \\
\hline VE2 & 35 & 9 & 13 & 22 & 47 & 35 & 49 & 4314 & 233 & $\mathrm{~L}$ & $L$ & L & $L$ \\
\hline VE3 & 82 & 9 & 13 & 22 & 47 & 6 & 69 & 4180 & 224 & $L$ & $H$ & $\mathrm{H}$ & $P$ \\
\hline VE4 & 92 & 9 & 13 & 22 & 47 & 66 & 87 & 4431 & 219 & $\mathrm{H}$ & $\mathrm{H}$ & $\mathbf{L}$ & N \\
\hline VE5 & 102 & 9 & 13 & 22 & 47 & 9 & 11 & 4977 & 191 & ML & $\mathrm{N}$ & ML & L \\
\hline VE6 & 102 & 8 & 13 & 22 & 47. & 41 & 97 & 3554 & 185 & ML & $\mathbf{H}$ & $\mathrm{N}$ & $\mathbf{N}$ \\
\hline VE7 & 102 & 21 & 13 & 22 & 47 & 9 & 92 & 3859 & 228 & $\mathrm{H}$ & $\mathbf{H}$ & $\mathbf{N}$ & ML \\
\hline VE8 & 92 & 21 & 87 & 22 & 47 & 7 & 38 & 2783 & 248 & L & $M L$ & ML & $\mathrm{H}$ \\
\hline VE9 & 102 & 71 & 87 & 22 & 47 & 18 & 84 & 2506 & 243 & L & $\mathrm{H}$ & $\mathrm{ML}$ & $\underline{L}$ \\
\hline VE10 & 74 & 21 & 48 & 22 & 47 & 43 & 50 & 3156 & 179 & $\mathrm{H}$ & $\mathrm{H}$ & $\mathrm{ML}$ & $\mathrm{H}$ \\
\hline VE11 & 92 & 21 & 48 & 22 & 47 & 11 & 92 & 3255 & 195 & $\underline{L}$ & $\mathrm{H}$ & $P$ & $\mathrm{ML}$ \\
\hline VE12 & 92 & 9 & 48 & 22 & 47 & 14 & 51 & 2405 & 294 & $\mathrm{~N}$ & L & $\mathbf{P}$ & $\mathrm{ML}$ \\
\hline$V E 13$ & 35 & 8 & 48 & 22 & 47 & 38 & 47 & 2464 & 226 & ML & $L$ & $L$ & $P$ \\
\hline VE14 & 92 & 71 & 48 & 22 & 47 & 24 & 84 & 2336 & 233 & ML & $M L$ & ML & $L$ \\
\hline
\end{tabular}

To illustrate the MADM techniques here we only use one variable of each type to rank the alternatives (price, cost and trust).

\section{Linguistic 2-tuples approach}

- First we have normalized the numerical and interval variables, then applied the membership function to transform them into fuzzy sets, composed by 5 fuzzy numbers according to the cardinality previously chosen. After the information has been unified, we have transformed the fuzzy sets in linguistic 2-tuples, and then, we have calculated the aggregate grade evaluation (through an arithmetic mean operator). The membership function considered is:

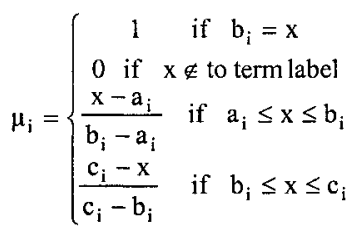


Table 3: Fuzzy sets, linguistic 2-tuples and the dominance degree

\begin{tabular}{|c|c|c|c|c|c|c|c|}
\hline & price & cost & trust & price & cost & trust & $\begin{array}{l}\text { aggregate grade } \\
\text { evaluation }\end{array}$ \\
\hline VE1 & $(0.64,1,0.07,0,0)$ & $(0.37,0.63,0,0,0)$ & $(0,0,1,0,0)$ & $(\mathrm{VL},-0.33)$ & $(L,-0.37)$ & $(M L, 0)$ & $(N L, 0.1)$ \\
\hline VE2 & $(0,0,59,0,98,0,0)$ & $(0,1,0,0,0)$ & $(0,1,0,0,0)$ & $(\mathrm{ML},-0.37)$ & $(L, 0)$ & $(L, 0)$ & (VL, 0.21$)$ \\
\hline VE3 & $(0.74,1,1,0.75,0)$ & $(0,0,79,0.21,0,0)$ & $(0,1,0,0,0)$ & $(M L,-0.5)$ & $(L, 0.21)$ & $(L, 0)$ & $(M, 0.24)$ \\
\hline VE4 & $(0,0,0.37,1,0.48)$ & $(0,17,0,83,0,0,0)$ & $(0,0,0,1,0)$ & $(\mathrm{VH}, 0.06)$ & $\left(L_{+}-0.17\right)$ & $(H, 0)$ & (ML,0.29) \\
\hline VE5 & $(0.63,0.45,0,0,0)$ & $(1,0,0,0,0)$ & $(0,0,1,0,0)$ & $(\mathrm{N}, 0.42)$ & $(\mathrm{VL}, 0)$ & $(M L, 0)$ & $(V L,-0.19)$ \\
\hline VE6 & $(0,0.37,1,1,0.86)$ & $(0,0,0.84,0.16,0)$ & $(0,0,1,0,0)$ & $(\mathrm{VH},-0.27)$ & $(M L, 0,16)$ & $\langle\mathrm{ML}, 0\rangle$ & $(M L, 0.3)$ \\
\hline VE7 & $(0.64,1,1,1,0.69)$ & $(0,0.31,0.69,0,0)$ & $(0,0,0,1,0)$ & $(\mathrm{ML}, 0.02)$ & $(M L-0.31)$ & $(H, 0)$ & $(M L, 0.24)$ \\
\hline VE8 & $(0.74,1,0.51,0,0)$ & $(0,0,0,0.68,0.32)$ & $(0,1,0,0,0)$ & $(\mathrm{ML},-0.1)$ & $(\mathrm{VH}, 0.32)$ & $(L, 0)$ & $(M L,-0,26)$ \\
\hline VES & $(0.27,1,1,1,0.34)$ & $(0,0,0,0.26,0,74)$ & $(0,1,0,0,0)$ & $(M L, 0.04)$ & $(P,-0.26)$ & $(L, O)$ & $(M L, 0,26)$ \\
\hline VE10 & $(0,0,29,1,0,0)$ & $(0,0,0.24,0,76,0)$ & $(0,0,0,1,0)$ & $(M L,-0.23)$ & $(\mathrm{NH},-0,24)$ & $(H, 0)$ & $(\mathrm{NH},-0,49)$ \\
\hline VE11 & $(0.56,1,1,1,0.70)$ & $(0,0,0.39,0.61,0)$ & $(0,1,0,0,0)$ & $(M L, 0.07)$ & $(\mathrm{VH},-0.39)$ & $(\mathrm{L}, 0)$ & $(M L,-0.11)$ \\
\hline VE12 & $(0.44,1,0.04,0,0)$ & $(0,0,0,0.1,0.9)$ & $(1,0,0,0,0)$ & $(V L,-0.27)$ & $(P,-0.1)$ & $(N, 0)$ & $(M L,-0.46)$ \\
\hline VE13 & $(0,0.47,0.89,0,0)$ & $(0,0,0,0.19,0.81)$ & $(0,0,1,0,0)$ & $(M L,-0.34)$ & $(P,-0.19)$ & $(M L, 0)$ & $(M L, 0.49)$ \\
\hline VE14 & $(0.06,1,1,1,0,35)$ & $(0,0,0,0,1)$ & $(0,0,1,0,0)$ & (ML, 0.17$)$ & $(P, 0)$ & $(M L, 0)$ & $(\mathrm{NH},-0.28)$ \\
\hline
\end{tabular}

The results obtained suggest that the group of companies chosen to carry out the project should be those in alternative VE14, because this is the one with higher linguistic label ( $\mathrm{VH}$ - very high) and with the less negative value of the symbolic translation $(-0,28)$.

\section{Distance approach}

Distance measures formulas:

- For interval numbers (Tran and Duckstein, 2002)

$$
D(A, B)=\sqrt{\left[\left(\frac{a_{1}+a_{2}}{2}\right)-\left(\frac{b_{1}+b_{2}}{2}\right)\right]^{2}+\frac{1}{3}\left[\left(\frac{a_{2}-a_{1}}{2}\right)^{2}+\left(\frac{b_{2}-b_{1}}{2}\right)^{2}\right]}
$$

- For triangular fuzzy numbers (Li and Yang, 2004)

Let $A=\left(a_{1}, a_{2}, a_{3}\right)$ and $B=\left(b_{1}, b_{2}, b_{3}\right)$ be two triangular fuzzy numbers.

$D(A, B)=\sqrt{\frac{1}{3}\left[\left(a_{1}-b_{1}\right)^{2}+\left(a_{2}-b_{2}\right)^{2}+\left(a_{3}-b_{3}\right)^{2}\right]}$

- For real numbers

Let $A$ and $B$ be two real numbers

$$
D(A, B)=\sqrt{(a-b)^{2}}
$$

Table 2: Normalized distances

\begin{tabular}{|c|c|c|c|c|c|c|c|c|}
\hline \multirow{2}{*}{ alternatives } & \multicolumn{2}{|c|}{ price } & \multicolumn{2}{c|}{ cost } & \multicolumn{2}{c|}{ trust } & \multicolumn{2}{c|}{ average } \\
\cline { 2 - 10 } & $\max$ & $\min$ & $\max$ & $\min$ & $\max$ & $\min$ & $\max$ & $\min$ \\
\hline VE1 & 0,38 & 0,15 & 0,84 & 0,16 & 0,43 & 0,43 & 0,55 & 0,25 \\
\hline VE2 & 0,71 & 0,25 & 0,75 & 0,25 & 0,68 & 0,20 & 0,71 & 0,23 \\
\hline VE3 & 0,51 & 0,29 & 0,70 & 0,30 & 0,68 & 0,20 & 0,63 & 0,27 \\
\hline VE4 & 1,00 & 0,20 & 0,79 & 0,21 & 0,20 & 0,68 & 0,67 & 0,36 \\
\hline VE5 & 0,00 & 0,00 & 1,00 & 0,00 & 0,43 & 0,43 & 0,48 & 0,14 \\
\hline VE6 & 0,91 & 0,53 & 0,46 & 0,54 & 0,43 & 0,43 & 0,60 & 0,50 \\
\hline VE7 & 0,63 & 0,41 & 0,58 & 0,42 & 0,20 & 0,68 & 0,47 & 0,50 \\
\hline VE8 & 0,25 & 0,84 & 0,17 & 0,83 & 0,68 & 0,20 & 0,37 & 0,62 \\
\hline VE9 & 0,72 & 0,93 & 0,06 & 0,94 & 0,68 & 0,20 & 0,49 & 0,69 \\
\hline VE10 & 0,79 & 0,69 & 0,31 & 0,69 & 0,20 & 0,68 & 0,44 & 0,69 \\
\hline VE11 & 0,66 & 0,65 & 0,35 & 0,65 & 0,68 & 0,20 & 0,56 & 0,50 \\
\hline VE12 & 0,47 & 0,98 & 0,03 & 0,97 & 0,84 & 0,00 & 0,45 & 0,65 \\
\hline VE13 & 0,72 & 0,96 & 0,05 & 0,95 & 0,43 & 0,43 & 0,40 & 0,78 \\
\hline VE14 & 0,78 & 1,00 & 0,00 & 1,00 & 0,43 & 0,43 & 0,40 & 0,81 \\
\hline
\end{tabular}


The DM is now in a position to choose one particular alternative among these fourteen (potential VEs), according to his preferences: smallest distance to the ideal maximum (1) or the highest to the ideal minimum (0). If the ideal maximum is the purpose, VE8 is chosen ( 0,37 - distance to maximum) but, if it is the ideal minimum, VE14 is chosen $(0,81$ - distance to minimum). In case that the DM wants to take into consideration both points, the ranking order of all alternatives can be obtained by calculating the amplitude between the two average distance measures, maximum and minimum, and so VE14 may be considered the best one $(0,41$ - higher amplitude distance). Generally the rank order found is subjective and depends of the DM judgements. The linguistic 2-tuples approach has as major advantage (when compared with the distance measures approach) the fact that it presents a precise rank order to the DM.

\section{CONCLUSIONS}

The selection of partners is a critical issue in the formation of a VE, i.e. the choice of entities to be involved in an emergent business opportunity, according to their attributes and interactions. This work is a first step in the development of an integrated DSS capable of handling this problem. Moreover such a DSS will hopefully become a simulation tool encouraging informal cooperation between the companies of the network. The system provides the necessary support to structure the VE formation (or re-organization) problem, allows the existence of different types of data (numerical, linguistic, binary, interval), individual and group decision makers, and performs several MADM techniques in order to generate a ranking list of potential VEs. It allows the analysis, for a given time horizon, of how the network reacts when various projects take place, simultaneously or not, and it enables different types of "what if" analysis. The system was designed in a modular way, allowing an easy incorporation of different metaheuristics for the generation of alternatives, or other MADM techniques for supporting the ranking and choice of those alternatives.

\section{REFERENCES}

1. Katzy BR, Dissel M. A toolset for building the virtual enterprise. Journal of Intelligent Manufacturing 2001; 12, 2: 121-131

2. Camarinha-Matos LM Hamideh Afsarmanesh. Elements of a base VE infrastructure. Computers in Industry $2003 ; 51: 139-163$.

3. Scheubreina R, Ziontsb S. A problem structuring front end for a multiple criteria decision support system. Computers \& Operations Research, In press, Corrected Proof, Available online 16 july 2004.

4. Climaco J, ed. "Multicriteria analysis". New York: Springer-Verlag, 1997.

5. Zanakis SH, Solomon A, Wishart N, Dublish D. Multi-attribute decision making: a simulation comparison of select methods. European Journal of Operations Research 1998; 107, 3: 507-529.

6. Pohekar SD, Ramachandran M. Application of multi-criteria decision making to sustainable energy planning-A review. Renewable and Sustainable Energy Reviews 2004; 8: 365-381.

7. Li Y, Xiuwu, L. Decision support for risk analysis on dynamic alliance. Decision Support Systems, In press, Corrected Proof, Available online 18 December 2004.

8. Fan Z-P, Hu G-F, Xiao S-H. A method for multiple attribute decision-making with the fuzzy preference relation on alternatives. Computers \& Industrial Engineering 2004; 46: 321-327. 
9. Reeves CR, ed. "Modern Heuristic Techniques for Combinatorial Problems", John Wiley \& Sons, Inc. NY, USA, 1993.

10. Osman IH, Kelly JP. "Meta-Heuristics: An Overview. In Meta-Heuristics: Theory and Applications, Osman IH, Kelly JP ed. Kluwer Academic Publishers. Boston, USA, 1996.

11. Glover F. Tabu Search, Part I. ORSA Journal on Computing 1989; 1: 190-206.

12. Glover F. Tabu Search, Part II. ORSA Journal on Computing 1990; 2: 4-32.

13. Glover F. A user's guide to tabu search. Annals of Operations Research 1993; 41: 3-28.

14. Laguna M, Glover F. What is Tabu Search. Colorado Business Review 1996; 61: 5-12.

15. Glover F, Laguna M. "Tabu search". Kluwer Academic Publishers. Boston, USA, 1997.

16. Herrera F, Martinez L, Sanchez PJ. Decision Aiding Managing non-homogeneous information in group decision making. European Journal of Operational Research 2004; 166, 1: I15-132.

17. Herrera F, Herrera-Viedma E. Linguistic decision analysis: Steps for solving decision problems under linguistic information. Fuzzy Sets and Systems 2000; 115: 67-82.

18. Delgado M, Vila MA, Voxman W. On a canonical representation of fuzzy numbers. Fuzzy Sets and Systems 1998; 93: 125-135.

19. Herrera F, Lopez E, Rodriguez MA. A linguistic decision model for promotion mix management solved with genetic algorithms. Fuzzy Sets and Systems 2002; 131:47-61.

20. Herrera F, Martinez L. A 2-tuple fuzzy linguistic representation model for computing with words. IEEE Transactions on Fuzzy Systems 2000; 8, 6:746-752.

21. Degani $R$, Bortolan $G$. The problem of linguistic approximation in clinical decision making. International Journal of Approximate Reasoning 1998; 2: 143-162.

22. Herrera F, Herrera-Viedma, E, Martínez L. A fusion approach for managing multi-granularity linguistic term sets in decision making. Fuzzy Sets and Systems 2000; 114: 43-58.

23. Xu Z. A method based on linguistic aggregation operators for group decision making with linguistic preference relations. Information Sciences 2004; 166, 1-4: 19-30.

24. Ding J-F, Liang G-S. Using fuzzy MCDM to select partners of strategic alliances for liner shipping. Information Sciences. In press, Corrected Proof, Available online 28 August 2004.

25. Butler J, Jia J, Dyer J. Simulation techniques for the sensitivity analysis of multi-criteria decision models. European Journal of Operations Research 1997; 103: 531-546. 